\title{
Balloon-assisted thoracoscopic surgery for apex posterior mediastinal benign neurogenic tumor
}

\author{
Xiaohui Xu, Xiaoyun Zhou, Chao Gao, Yushang Cui \\ Department of Thoracic Surgery, Peking Union Medical College Hospital, Beijing 100730, China \\ Correspondence to: Yushang Cui, Department of Thoracic Surgery, Peking Union Medical College Hospital, Beijing 100730, China. \\ Email: cuiyushang@126.com.
}

Submitted Aug 08, 2019. Accepted for publication Aug 30, 2019.

doi: $10.21037 /$ jtd.2019.09.08

View this article at: http://dx.doi.org/10.21037/jtd.2019.09.08

\section{Introduction}

Improvement in technique and instrumentation makes thoracoscopic surgery increasingly popular in the thoracic area, including posterior mediastinal benign neurogenic tumor (PMBNT), which was one of the earliest tumors resected by thoracoscopic surgery. PMBNT is a common thoracic tumor. Neurogenic tumors account for $75 \%$ of posterior mediastinal tumors (1), in which $70-80 \%$ are benign in adults $(2,3)$. Currently, thoracoscopic resection has been widely used in the treatment of PMBNT, and some surgeons even successfully have performed single-port thoracoscopic resection. However, the high rate of Horner's syndrome after apex PMBNT resection poses a surgical challenge due to the complex regional anatomy. Apex PMBNT locates at the T1 and T2 vertebral level, involving important nerves such as the sympathetic chain at T1 level. The injury or denervation of sympathetic chain at T1 level by energy sources is a major cause of Horner's syndrome. Sympathetic chain is easily to be hurt during the resection of apex PMBNT due to the narrow confines of the thoracic inlet. In addition, emergency hemostasis of intercostal arterial hemorrhage or other bleeding by electrocautery burn can also lead to irreversible damage of sympathetic chain. Therefore, posterior mediastinal tumor in apex thorax, tumor size over $6 \mathrm{~cm}$ in diameter, and involvement of the spinal cord by dumbbell tumors once have been considered as the three major obstacles of thoracoscopic surgery for treating posterior mediastinal tumor $(4,5)$. For PMBNT located below the T3 or T4 vertebral level, the first step of resection is separating the peripheral connection of the tumor using electrically and ultrasonically activated scalpels, followed by lifting the tumor from the primary position, and then removing it. However, the same approach can cause high incidence of postoperative complication for apex PMBNT because of limited anatomic space. Horner's syndrome has been reported in $10 \%$ to $20 \%$ patients with PMBNT at different vertebral levels in some studies with large sample size. However, the above-mentioned incidence may be underestimated since approximately $95 \%$ of posterior mediastinal masses arise from the intercostal nerve rami or the sympathetic chain region $(6,7)$. The incidence of Horner's syndrome could increase by 6 times to more than $60 \%$ for tumors in apex thorax with the assumption that the posterior mediastinal tumors are evenly distributed at each intercostal level and the apex PMBNT account for about one sixth of the overall PMBNT population. Horner's syndrome is an unacceptable complication that significantly influences the quality of life for patients, especially for those who are asymptomatic at presentation (8), which remains an unmet medical need. From Jan 2017 to Dec 2018, Yushang Cui and his colleagues successfully performed apex PMBNT resection using balloon dilation to separate tumor from peripheral tissues in four cases with schwannomas located at T1/T2 level, no Horner's syndrome was observed and all patients recovered well. Here we describe the key points of balloon dilation in the apex PMBNT resection based on our experience.

\section{Operative techniques}

We defined the furthest point and nearest point of a spherical tumor as the distal pole and the proximal pole, respectively, under the thoracoscopic vision. Thoracoscopic surgery for apex thoracic tumor is limited by the small size 


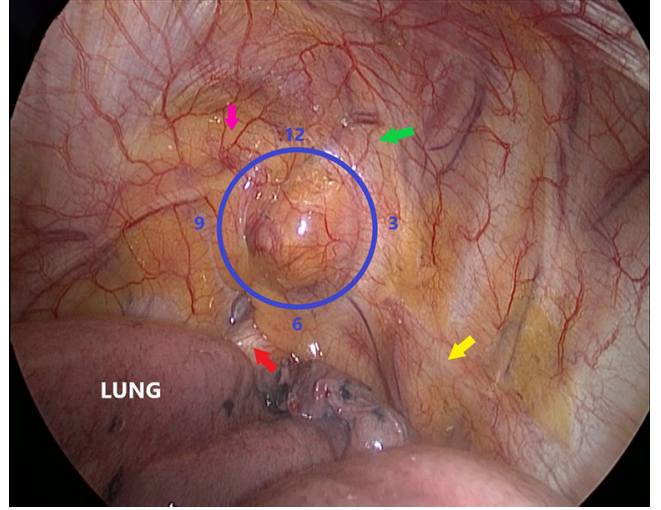

Figure 1 Left apex posterior mediastinal benign neurogenic tumor (PMBNT). Yellow arrow, sympathetic chain; Green arrow: $2^{\text {nd }}$ rib; Pink arrow: $1^{\text {st }}$ rib; Red arrow: left subclavian artery; blue circle with numerals: dial.

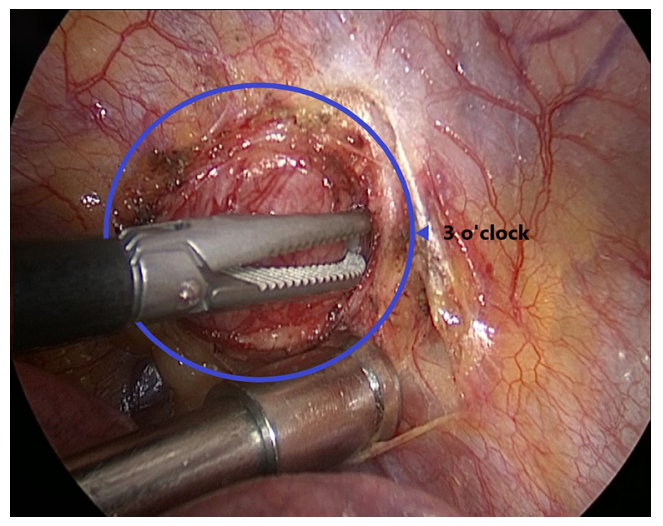

Figure 2 Separating 3 o'clock position. Blue circle with numerals: dial.

of the utility port since tumors at the thoracic apex are adjacent to important structures including blood vessels, nerves, and organs (Figure 1). Our surgical procedure was performed under general anesthesia with doublelumen intubation. The patient was placed in the left lateral decubitus position and slightly tilted towards prone position, with the operative table tilted anteriorly. A $1-\mathrm{cm}$ incision for thoracoscopic port was made at the sixth intercostal space (ICS) in the mid-axillary line. A single incision $(2.5 \mathrm{~cm})$ was then made in the anterior axillary line in the third ICS. As the tumor is located on the lateral side of $\mathrm{T} 1$ and $\mathrm{T} 2$ vertebrae where the sympathetic chain passes, we identified the location where tumor involved the sympathetic chain by following the normal sympathetic chain from the surface of the $\mathrm{T} 3$ or $\mathrm{T} 4$ vertebrae, and electrocautery should be

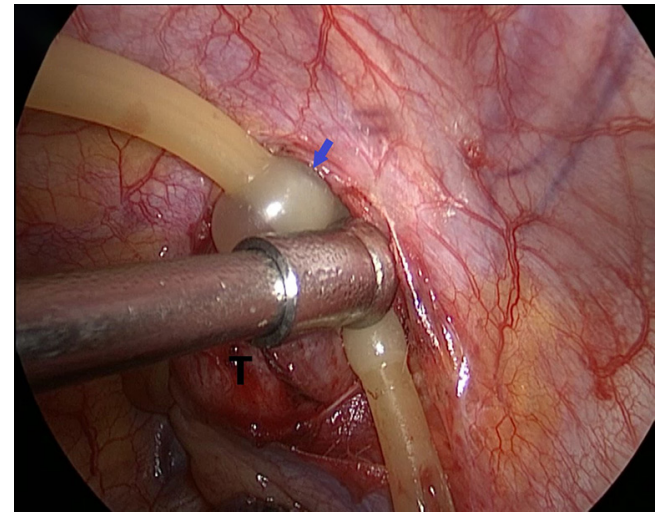

Figure 3 Injecting water into balloon at 3 o'clock position. T: tumor; blue arrow: balloon.

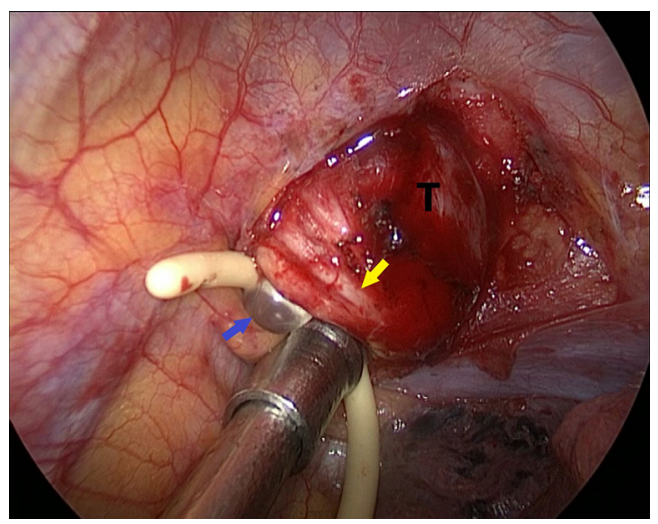

Figure 4 Injecting water into balloon at 9 o'clock position. T: tumor; blue arrow: balloon; yellow arrow: sympathetic chain at $1^{\text {st }}$ vertebrae level.

avoided in this area. The mediastinal pleura and the outer membrane of the tumor was opened $2-3 \mathrm{~mm}$ away from the base of tumor to avoid damage to the nerves. We first separated the posterolateral side of the tumor (3 o'clock position) by forceps since there is no important structure except the first rib medially (Figure 2). A balloon catheter of $8 \#$ was then placed in this $2-\mathrm{mm}$ gap and injected with water (Figure 3). Adequate pressure should be maintained for the balloon to expand in the gap without intruding into the thoracic cavity, which prevented balloon bursting and reduction of the dissecting effect of balloon dilation in the gap by pressure leakage. The balloon was kept in position for 1 minute, after which it was collapsed and removed. Repeat the abovementioned procedures at 9 o'clock position of the tumor (Figure 4). We then changed catheter from $8 \#$ to $10 \#$ and even to $12 \#$ to widen and deepen the 


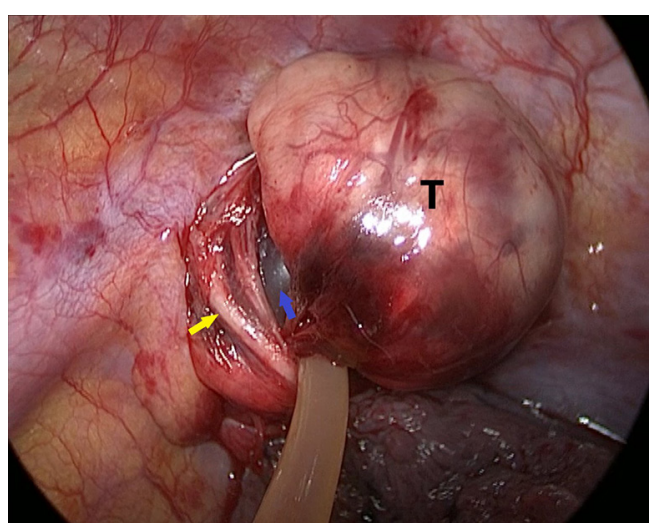

Figure 5 Extruding the tumor into the chest cavity from original position. T: tumor; Blue arrow: balloon; Yellow arrow: sympathetic chain at $1^{\text {st }}$ vertebrae level.

gap at 3 and 9 o'clock position until the gap extended to the distal pole. A balloon catheter larger than all previous ones was inserted into the gap close to the distal pole, extruding the tumor into the chest cavity from apex thorax (Figure 5). A complete resection was then performed without using energy sources.

\section{Comments}

It is essential to protect sympathetic chain from permanent Horner's syndrome in this procedure. The first step is to identify the binding point of the sympathetic chain and the tumor. We employed balloon dilation to widen and deepen the gap surrounding the tumor at a safe distance from the binding point. This procedure was repeated with larger balloon catheters at 3 and 9 o'clock position, providing a wide corridor to the distal pole. Finally, the tumor was extruded from the original position by a larger balloon with more pressure. This technique is developed on the basis of the characteristics of benign tumor (homogeneous, encapsulated, and well circumscribed with space to adjacent structure) and the Pascal's Law (in a fluid at rest in a closed container, a pressure change in one part is transmitted without loss to every portion of the fluid and to the walls of the container), which provides a safe procedure with reduced injury to nerves and vessels, therefore preventing severe hemorrhage and conversion to thoracotomy.

In summary, apex PMBNT could be successfully extruded without damage to sympathetic chain at T1 level through balloon dilation.

\section{Acknowledgments}

None.

\section{Footnote}

Conflicts of Interest: The authors have no conflicts of interest to declare.

Ethical Statement: The authors are accountable for all aspects of the work in ensuring that questions related to the accuracy or integrity of any part of the work are appropriately investigated and resolved. Written informed consent was obtained from the patient for publication of this manuscript and any accompanying images.

\section{References}

1. Davidson KG, Walbaum PR, McCormack RJ. Intrathoracic neural tumors. Thorax 1978;33:359-67.

2. Liu HP, Yim AP, Wan J, et al. Thoracoscopic removal of intrathoracic neurogenic tumors: a combined Chinese experience. Ann Surg 2000;232:187-90.

3. Silverman NA, Sabiston DC Jr. Mediastinal masses. Surg Clin North Am 1980;60:757-77.

4. Li Y, Wang J. Experience of video-assisted thoracoscopic resection for posterior mediastinal neurogenic tumours: a retrospective analysis of 58 patients. ANZ J Surg 2013;83:664-8.

5. Venissac N, Leo F, Hofman P, et al. Mediastinal neurogenic tumors and video-assisted thoracoscopy always the right choice? Surg Laparosc Endosc Percutan Tech 2004;14:20-2.

6. Reeder LB. Neurogenic tumors of the mediastinum. Semin Thorac Cardiovasc Surg 2000;12:261-7.

7. Kumar A, Kumar S, Aggarwal S, et al. Thoracoscopy: the preferred approach for the resection of selected posterior mediastinal tumors. J Laparoendosc Adv Surg Tech A 2002;12:345-53.

8. Abdel Rahman AR, Sedera MA, Mourad IA, et al. Posterior Mediastinal Tumors: Outcome of Surgery. J Egypt Natl Canc Inst 2005;17:1-8.

Cite this article as: Xu X, Zhou X, Gao C, Cui Y. Balloonassisted thoracoscopic surgery for apex posterior mediastinal benign neurogenic tumor. J Thorac Dis 2019;11(9):4018-4020. doi: 10.21037/jtd.2019.09.08 